\title{
A characterization of stochastic dominance efficiency
}

\author{
Haris Aziz
}

Received: 10 February 2014 / Accepted: 12 June 2014 / Published online: 1 July 2014

(C) Society for the Advancement of Economic Theory 2014

\begin{abstract}
Pareto optimality is one of the most central concepts in economics. SD-efficiency (efficiency with respect to stochastic dominance) is the most established generalization of Pareto optimality for randomized settings. In the last decade, a number of characterizations of SD-efficiency have been presented especially in the domain of assignments. We present a new characterization of SD-efficiency in the more general domain of randomized social choice.
\end{abstract}

Keywords Efficiency $\cdot$ Stochastic dominance $\cdot$ Social choice theory · Assignment problem

JEL Classification $\quad$ C70 $\cdot$ D61 $\cdot$ D71

\section{Introduction}

Pareto optimality has been termed the "single most important tool of normative economic analysis" Moulin (2003). In this paper, we consider stochastic dominance (SD)-efficiency that is arguably the canonical generalization of Pareto optimality in randomized settings (Abdulkadiroğlu and Sönmez 2003; Aziz et al. 2013a; Bogomolnaia and Moulin 2001; Carroll 2010; Katta and Sethuraman 2006; McLennan 2002; Manea 2008). SD-efficiency is efficiency with respect to the well-established SD lottery extension that extends preferences over alternatives to preferences over lotteries over alternatives. The relation SD is fundamental because one lottery stochastically dominates another one if and only if the former yields at least as much expected utility as the latter for any von-Neumann-Morgenstern utility representation consistent with

H. Aziz $(\bowtie)$

NICTA and UNSW, Sydney 2033, Australia

e-mail: haris.aziz@nicta.com.au 
the ordinal preferences (see e.g., Cho 2012). SD-efficiency was first used by Bogomolnaia and Moulin $(2001)^{1}$ and has received considerable interest within the domain of the random assignment problem in which agents express preferences over objects and the outcome is an assignment that assigns an object to each agent (Abdulkadiroğlu and Sönmez 2003; Bogomolnaia and Moulin 2001; Katta and Sethuraman 2006; McLennan 2002; Manea 2008). The random assignment setting is a subdomain of the randomized voting setting (see e.g., Aziz et al. 2013b; Carroll 2010; Gibbard 1977) in which each discrete assignment can be considered as an alternative and each agent is indifferent among all the discrete assignments in which he gets the same object.

Characterization of SD-efficiency has been an active area of research within economic theory. Bogomolnaia and Moulin (2001) and Katta and Sethuraman (2006) showed that a random assignment is SD-efficient if and only if a certain binary relation between objects is acyclic. Abdulkadiroğlu and Sönmez (2003) presented another interesting characterization of SD-efficient assignments based on a concept of domination defined over sets of assignments. McLennan (2002), Manea (2008) and Athanassoglou (2011) characterized SD-efficient assignments as ex-ante welfare maximizing for some vNM utilities compatible with the ordinal preferences. Carroll (2010) generalized the result to the setting of randomized social choice.

In this paper, we present a new characterization of SD-efficiency. We characterize SD-efficiency in the general randomized social choice setting as a property of the support of the outcomes. As a corollary, we show that SD-efficiency only depends on the support of the lottery and not the actual probabilities. In the charaterization, we show a formal connection between the SD lottery extension and the responsive set extension.

\section{Preliminaries}

\subsection{Social choice and lotteries}

Consider the social choice setting in which there is set of agents $N=\{1, \ldots, n\}$, a set of alternatives $A=\left\{a_{1}, \ldots, a_{m}\right\}$ and a preference profile $\succsim=\left(\succsim_{1}, \ldots, \succsim_{n}\right)$ such that each $\succsim_{i}$ is a complete and transitive relation over $A$. We have $a \succsim_{i} b$ denote that agent $i$ values alternative $a$ at least as much as alternative $b$ and write $\succ_{i}$ for the strict part of $\succsim_{i}$, i.e., $a \succ_{i} b$ if $a \succsim_{i} b$ but not $b \succsim_{i} a$. Furthermore, $\sim_{i}$ denotes $i$ 's indifference relation, i.e., $a \sim_{i} b$ if both $a \succsim_{i} b$ and $b \succsim_{i} a$. Whenever all $\succsim_{i}$ are antisymmetric, we say that agents have strict preferences. The relation $\succsim_{i}$ results in equivalence classes $E_{i}^{1}, E_{i}^{2}, \ldots, E_{i}^{k_{i}}$ for some $k_{i}$ such that agent $i$ is indifferent among all alternatives in the same equivalence class and $a \succ_{i} a^{\prime}$ for $a \in E_{i}^{l}$ and $a^{\prime} \in E_{i}^{l^{\prime}}$ for $l<l^{\prime}$. For example, if the preferences of agent 1 over alternative set $\{a, b, c\}$ are $a \sim_{1} b \succ_{1} c$, then $E_{1}^{1}=\{a, b\}, E_{1}^{2}=\{c\}$ and $k_{1}=2$.

\footnotetext{
1 Bogomolnaia and Moulin (2001) used the term ordinal efficiency for SD-efficiency. We use the latter term as used by Cho (2012) and Thomson (2013).
} 
Let $\Delta(A)$ denote the set of all lotteries (or probability distributions) over $A$. The support of a lottery $p \in \Delta(A)$, denoted by $\operatorname{supp}(p)$, is the set of all alternatives to which $p$ assigns a positive probability, i.e., $\operatorname{supp}(p)=\{x \in A \mid p(x)>0\}$. The probability assigned to alternative $a \in A$ by lottery $p$ is denoted by $p(a)$.

\subsection{Stochastic dominance}

To reason about lotteries, we need to reason about how agents compare lotteries. A lottery extension maps preferences over alternatives to (possibly incomplete) preferences over lotteries.

Given $\succsim_{i}$ over $A$, one can extend $\succsim_{i}$ to $\succsim_{i}^{\mathcal{E}}$ over $\Delta(A)$ where $\succsim_{i}^{\mathcal{E}}$ is a relation between lotteries based on lottery extension $\mathcal{E}$. We now define $\mathrm{SD}$ (stochastic dominance) which is a prominent lottery extension. Consider two lotteries $p, q \in \Delta(A)$. Stochastic dominance (SD) requires that for each alternative $x \in A$, the probability that $p$ selects an alternative that is at least as preferred as $x$ by the agent is greater or equal to the probability that $q$ selects such an alternative:

$$
p \succsim_{i}^{\mathrm{SD}} q \Longleftrightarrow \forall y \in A: \sum_{x \in A: x \succsim_{i} y} p(x) \geq \sum_{x \in A: x \succsim_{i} y} q(x)
$$

We say that lottery $p$ SD-dominates lottery $q$ if $p \succsim_{i}^{\text {SD }} q$ for all $i \in N$. We say that lottery $p$ strictly SD-dominates lottery $q$ if $p \succsim_{i}^{\mathrm{SD}} q$ for all $i \in N$ and $\neg\left(q \succsim_{i}^{\mathrm{SD}} p\right)$ for some $i \in N$.

\subsection{Efficiency}

A lottery $p \in \Delta(A)$ is SD-efficient if there exists no lottery $q$ such that $q \succsim_{i}^{\mathrm{SD}} p$ for all $i \in N$ and $q \succ_{i}^{\text {SD }} p$ for some $i \in N$. In other words, lottery $p$ is SD-efficient if it is not strictly SD-dominated. An alternative $a \in A$ is Pareto optimal if there exists no alternative $b$ such that $b \succsim_{i} a$ for all $i \in N$ and $b \succ_{i} a$ for some $i \in N$. A lottery is $e x$ post efficient if it is a lottery over Pareto optimal alternatives. SD-efficiency is strictly stronger than ex post efficiency (Bogomolnaia and Moulin 2001).

Example 1 (Stochastic dominance) Consider the two agent setting in which the alternative set $A$ is $\{a, b, c\}$, the preferences of agent 1 are $a \sim_{1} b \succ_{1} c$ and the preferences of agent 2 are $a \succ_{2} b \succ_{2} c$. Consider lottery $p$ such that $p(a)=2 / 3$ and $p(c)=1 / 3$ and lottery $q$ such that $q(a)=1$. Then, $q$ strictly SD-dominates $p$. Hence, $p$ is not SD-efficient.

\section{Characterizing SD-efficiency}

We characterize SD-efficiency. SD-efficient outcomes have been characterized in a number of ways in the restricted setting of the assignment problems (Athanassoglou 2011; Bogomolnaia and Moulin 2001; Katta and Sethuraman 2006; Manea 2008; 
McLennan 2002). Our characterization is for general social choice. We first define the responsive set extension (see e.g., Barberà et al. 2004) that lifts preferences over alternatives to preferences over sets of alternatives. For the definition, we use the notion of a multi-set which is a generalization of the notion of a set in which members are allowed to appear more than once.

Definition 1 (Responsive set extension) Consider two multi-sets $S$ and $T$ with elements from $A$. Then, $S \succsim_{i}^{\mathrm{RS}} T$ i.e., $S$ is weakly preferred by $i$ via the responsive set extension iff there exists a bijection $f_{i}: T \rightarrow S$ such that for each $a \in T, f_{i}(a) \succsim_{i} a$.

Definition 2 (Dominated sub-support) The support of a lottery $p$ admits a dominated sub-support if there exists a multi-set $T$ with all elements from $\operatorname{supp}(p)$ and a multi-set $S$ with elements from $A$ for which $S \succsim_{i}^{\mathrm{RS}} T$ for all $i \in N$ and $S \succ_{i}^{R S} T$ for some $i \in N$.

Example 2 (Responsive set extension and dominated sub-support) Consider the two agent setting in which the alternative set $A$ is $\{a, b, c\}$, the preferences of agent 1 are $a \sim_{1} b \succ_{1} c$ and the preferences of agent 2 are $a \succ_{2} b \succ_{2} c$. The responsive set extension extends the preferences of agent 1 over multi-sets of size two consisting of elements of $A$ as follows.

$$
\{a, a\} \sim_{1}^{\mathrm{RS}}\{a, b\} \sim_{1}^{\mathrm{RS}}\{b, b\} \succ_{1}^{\mathrm{RS}}\{a, c\} \sim_{1}^{\mathrm{RS}}\{b, c\} \succ_{2}^{\mathrm{RS}}\{c, c\} .
$$

The responsive set extension extends the preferences of agent 2 over multi-sets of size two consisting of elements of $A$ as follows.

$$
\begin{aligned}
&\{a, a\} \succ_{2}^{\mathrm{RS}}\{a, b\} \succ_{2}^{\mathrm{RS}}\{b, b\} \succ_{2}^{\mathrm{RS}}\{b, c\} \succ_{2}^{\mathrm{RS}}\{c, c\} . \\
&\{a, b\} \succ_{2}^{\mathrm{RS}}\{a, c\} \succ_{2}^{\mathrm{RS}}\{b, c\} .
\end{aligned}
$$

Consider lottery $p$ such that $p(a)=2 / 3$ and $p(c)=1 / 3$. Then, $\operatorname{supp}(p)=$ $\{a, c\}$. We note that there exists a multi-set $\{a, a\}$ with elements from $A$ for which $\{a, a\} \succ_{i}^{\mathrm{RS}}\{a, c\}$ for all $i \in\{1,2\}$. Hence, $p$ has a dominated sub-support.

Lemma 1 For a lottery $p$, if $\operatorname{supp}(p)$ admits a dominated sub-support, then $p$ is not SD-efficient.

Proof Let us assume that $\operatorname{supp}(p)$ admits a dominated sub-support. Then there exists some multi-set $T$ with elements from $\operatorname{supp}(p)$ for which there is a multi-set $S$ such that $|S|=|T|$ and $S \succsim_{i}^{\mathrm{RS}} T$ for all $i \in N$ and $S \succ_{i}^{\mathrm{RS}} T$ for some $i \in N$.

By the definition of responsiveness, for each $i \in N$ there exists a bijection $f_{i}$ : $T \rightarrow S$ such that for each $a \in T, f_{i}(a) \succsim_{i} a$. Now, for $p$ and some arbitrarily small $\epsilon>0$, let lottery $q$ be such that for each $a \in A, q(a)=p(a)-\epsilon($ \#copies of $a$ in $T)+$ $\epsilon$ (\#copies of $a$ in $S$ ). For each $i$, an $\epsilon$ decrease in the probability of an alternative $a$ results in an $\epsilon$ increase in some alternative $f_{i}(a) \succsim_{i} a$. Therefore, $q \succsim_{i}^{\mathrm{SD}} p$ for all $i \in N$ and $q \succ_{i}^{\mathrm{SD}} p$ for some $i \in N$.

A lottery $p$ is rational if $p(a)$ is rational for all $a \in A$. A lottery is irrational if $p(a)$ is irrational for some $a \in A$. 
Lemma 2 If a rational lottery $p$ is not SD-efficient, then $\operatorname{supp}(p)$ admits a dominated sub-support.

Proof Let $p$ be a rational lottery that is not SD-efficient. Since $p$ is rational, $p(a)$ is rational for each $a \in A$. Since $p$ is not SD-efficient, there exists another lottery such that $q \succsim_{i}^{\mathrm{SD}} p$ for all $i \in N$ and $q \succ_{i}^{\mathrm{SD}} p$ for some $i \in N$. In particular, $q$ can be obtained as a solution of the following linear program:

$$
\begin{gathered}
\max \sum_{i \in N} \sum_{l=1}^{k_{i}} \sum_{a \in \bigcup_{k=1}^{l} E_{i}^{k}} q(a) \text { s.t. } \\
\sum_{a \in \bigcup_{k=1}^{l} E_{i}^{k}} q(a) \geq \sum_{a \in \bigcup_{k=1}^{l} E_{i}^{k}} p(a) \text { for each } i \in N \text { and } l \in\left\{1, \ldots, k_{i}\right\} \\
\\
\sum_{a \in A} q(a)=1 .
\end{gathered}
$$

Since the linear program has a finite optimum and the constants involved in the linear program are rational, therefore its solution is rational (Schrijver 1986). Hence, for each $a \in A, q(a)$ is rational. Since $q \neq p$, there exists a non-empty set of alternatives $C=\{c: q(c)<p(c)\}$. Similarly, there exists a non-empty set of alternatives $B=$ $\{b: q(b)>p(b)\}$. Since $p(c)>0$ for each $c \in C$, we know that $C \subseteq \operatorname{supp}(p)$.

Let $\delta=q-p$ so that $\delta(a)=q(a)-p(a)$ for all $a \in A$. Since $q(a)$ and $p(a)$ are both rational, hence $\delta(a)$ is also rational. The greatest common divisor of a set of rational numbers $X$ is the largest rational number that is a factor of all the members of set $X$. Let $\epsilon=G C D\{|\delta(a)|: a \in B \cup C\}$ where function $G C D$ returns the greatest common divisor of a set of rational numbers. We note that $\epsilon$ is well defined because it is the greatest common divisor of a set of positive rational numbers. Let $B^{\prime}$ be a multi-set of elements from $B$ where each $b \in B$ features $\delta(b) / \epsilon$ times. Let $C^{\prime}$ be a multi-set of elements from $C$ where each $c \in C$ features $\delta(c) / \epsilon$ times. Therefore, $q$ is a lottery such that

$$
q(a)= \begin{cases}p(a) & \text { if } a \in A \backslash(B \cup C) \\ p(a)-(|\delta(a)| / \epsilon) \epsilon & \text { if } a \in C \\ p(a)+(|\delta(a)| / \epsilon) \epsilon & \text { if } a \in B .\end{cases}
$$

Since $q$ strictly SD-dominates $p$, for each $i \in N$, and $c \in C$ for which there is an $\epsilon$ decrease, there is a corresponding $b \in B$ such that $b \succsim_{i} c$ for which there is an $\epsilon$ increase in probability. This implies that $q$ can be obtained from $p$ by decrementing each alternative in $C^{\prime}$ by $\epsilon$ and incrementing each alternative in $B^{\prime}$ by $\epsilon$. Therefore, $B^{\prime} \succsim_{i}^{\mathrm{RS}} C^{\prime}$ for all $i \in N$ and $B^{\prime} \succ_{i}^{\mathrm{RS}} C^{\prime}$ for some $i \in N$. Hence, $\operatorname{supp}(p)$ admits a dominated sub-support.

Lemma 3 If a lottery $p^{\prime}$ is irrational and not SD-efficient, then there exists a rational lottery $p$ such that $\sup p(p)=\operatorname{supp}\left(p^{\prime}\right)$ and $p$ is not SD-efficient. 
Proof Consider a lottery $p^{\prime}$ that is irrational and not SD-efficient. Since $p^{\prime}$ is not SD-efficient, there exists a lottery $q^{\prime}$ such that

$$
\sum_{a \in \bigcup_{k=1}^{l} E_{i}^{k}} q^{\prime}(a) \geq \sum_{a \in \bigcup_{k=1}^{l} E_{i}^{k}} p^{\prime}(a)
$$

for each $i \in N$ and $l \in\left\{1, \ldots, k_{i}\right\}$ and

$$
\sum_{a \in \bigcup_{k=1}^{l} E_{i}^{k}} q^{\prime}(a)>\sum_{a \in \bigcup_{k=1}^{l} E_{i}^{k}} p^{\prime}(a)
$$

for some $i \in N$ and some $l \in\left\{1, \ldots, k_{i}\right\}$.

Let $\delta^{\prime}=q^{\prime}-p^{\prime}$ so that $\delta^{\prime}(a)=q^{\prime}(a)-p^{\prime}(a)$ for all $a \in A$. From (1) and (2), it follows that

$$
\sum_{a \in \bigcup_{k=1}^{l} E_{i}^{k}} \delta^{\prime}(a) \geq 0
$$

for each $i \in N$ and $l \in\left\{1, \ldots, k_{i}\right\}$ and

$$
\sum_{a \in \bigcup_{k=1}^{l} E_{i}^{k}} \delta^{\prime}(a)>0
$$

for some $i \in N$ and some $l \in\left\{1, \ldots, k_{i}\right\}$.

We show that if there exists an irrational lottery $p^{\prime}$ that is not SD-efficient, then there exists a rational lottery $p$ such that $\operatorname{supp}(p)=\operatorname{supp}\left(p^{\prime}\right)$ and $p$ is not SD-efficient. Lottery $p$ is defined as follows:

$$
p(a)= \begin{cases}1 /\left|\operatorname{supp}\left(p^{\prime}\right)\right| & \text { if } a \in \operatorname{supp}\left(p^{\prime}\right) \\ 0 & \text { if } a \notin \operatorname{supp}\left(p^{\prime}\right)\end{cases}
$$

Note that $\operatorname{supp}(p)=\operatorname{supp}\left(p^{\prime}\right)$ and that $p(a)$ is rational for each $a \in A$. We now show that $p$ is not SD-efficient. Consider $q$ such that $q=p+\epsilon \delta^{\prime}$ where $\epsilon>0$ is arbitrarily small so that $q(a) \geq 0$ for all $a \in A$. From (3) and (4), it follows that

$$
\sum_{a \in \bigcup_{k=1}^{l} E_{i}^{k}} \delta^{\prime}(a) \epsilon \geq 0
$$

for each $i \in N$ and $l \in\left\{1, \ldots, k_{i}\right\}$ and

$$
\sum_{a \in \bigcup_{k=1}^{l} E_{i}^{k}} \delta^{\prime}(a) \epsilon>0
$$


for some $i \in N$ and some $l \in\left\{1, \ldots, k_{i}\right\}$. Therefore,

$$
\sum_{a \in \bigcup_{k=1}^{l} E_{i}^{k}} q(a)=\sum_{a \in \bigcup_{k=1}^{l} E_{i}^{k}}\left(p(a)+\delta^{\prime}(a) \epsilon\right) \geq \sum_{a \in \bigcup_{k=1}^{l} E_{i}^{k}} p(a)
$$

for each $i \in N$ and $l \in\left\{1, \ldots, k_{i}\right\}$ and

$$
\sum_{a \in \bigcup_{k=1}^{l} E_{i}^{k}} q(a)=\sum_{a \in \bigcup_{k=1}^{l} E_{i}^{k}}\left(p(a)+\delta^{\prime}(a) \epsilon\right)>\sum_{a \in \bigcup_{k=1}^{l} E_{i}^{k}} p(a)
$$

for some $i \in N$ and some $l \in\left\{1, \ldots, k_{i}\right\}$. Hence $q \succsim_{i}^{\mathrm{SD}} p$ for all $i \in N$ and $q \succ_{i}^{\mathrm{SD}} p$ for some $i \in N$. Thus, $p$ is not SD-efficient.

Theorem 1 For social choice, the following conditions are equivalent:

(i) lottery $p$ is SD-efficient.

(ii) $\operatorname{supp}(p)$ does not admit a dominated sub-support.

Proof From Lemma 1, we know that for a lottery $p$, if $\operatorname{supp}(p)$ admits a dominated sub-support, then $p$ is not SD-efficient. Therefore, if lottery $p$ is SD-efficient, then $\operatorname{supp}(p)$ does not admit a dominated sub-support.

It remains to be shown that if $\operatorname{supp}(p)$ does not admit a dominated sub-support, then $p$ is SD-efficient. Let us assume that lottery $p$ is not SD-efficient. We show that $\operatorname{supp}(p)$ admits a dominated sub-support. If $p$ is rational, then by Lemma $2, \operatorname{supp}(p)$ admits a dominated sub-support. If $p$ is not rational, then by Lemma 3, there exists a rational lottery $p^{\prime \prime}$ that has the same support as $p$ and which is not SD-efficient. By Lemma 2, we know that $\operatorname{supp}\left(p^{\prime \prime}\right)$ admits a dominated sub-support. Since $\operatorname{supp}(p)=\operatorname{supp}\left(p^{\prime \prime}\right)$, $\operatorname{supp}(p)$ admits a dominated sub-support.

As a corollary, if a lottery $p$ is SD-efficient and there is another lottery $q$ such that $\operatorname{supp}(q) \subset \operatorname{supp}(p)$, then $q$ is also SD-efficient. The characterization above also shows that SD-efficiency depends solely on the support of the lottery.

Acknowledgments This material is based upon work supported by the Australian Government's Department of Broadband, Communications and the Digital Economy, the Australian Research Council, the Asian Office of Aerospace Research and Development through grant AOARD-124056. The author thanks Felix Brandt and Florian Brandl for a number of insightful discussions on the topic.

\section{References}

Abdulkadiroğlu, A., Sönmez, T.: Ordinal efficiency and dominated sets of assignments. J. Econ. Theory 112(1), 157-172 (2003)

Athanassoglou, S.: Efficiency under a combination of ordinal and cardinal information on preferences. J. Math. Econ. 47, 180-185 (2011)

Aziz, H., Brandt, F., Brill, M.: On the tradeoff between economic efficiency and strategyproofness in randomized social choice. In: Proceedings of the 12th international conference on autonomous agents and multi-agent systems (AAMAS), pp. 455-462. IFAAMAS (2013a) 
Aziz, H., Brandt F., Stursberg, P.: On popular random assignments. In: Vöcking, B. (ed.) Proceedings of the 6th International Symposium on Algorithmic Game Theory (SAGT). Lecture Notes in Computer Science (LNCS), vol. 8146, pp. 183-194. Springer (2013b)

Barberà, S., Bossert, W., Pattanaik, P.K.: Ranking sets of objects. In: Barberà, S., Hammond, P.J., Seidl, C. (eds.) Handbook of Utility Theory, vol. II, chapter 17, pp. 893-977. Kluwer Academic Publishers, Dordrecht (2004)

Bogomolnaia, A., Moulin, H.: A new solution to the random assignment problem. J. Econ. Theory 100(2), 295-328 (2001)

Carroll, G.: An efficiency theorem for incompletely known preferences. J. Econ. Theory 145(6), 2463-2470 (2010)

Cho, W.J.: Probabilistic assignment: a two-fold axiomatic approach. Unpublished manuscript (2012)

Gibbard, A.: Manipulation of schemes that mix voting with chance. Econometrica 45(3), 665-681 (1977)

Katta, A.-K., Sethuraman, J.: A solution to the random assignment problem on the full preference domain. J. Econ Theory 131(1), 231-250 (2006)

Manea, M.: Random serial dictatorship and ordinally efficient contracts. Int. J. Game Theory 36, 489-496 (2008)

McLennan, A.: Ordinal efficiency and the polyhedral separating hyperplane theorem. J. Econ Theory $\mathbf{1 0 5}(2)$, 435-449 (2002)

Moulin, H.: Fair Division and Collective Welfare. The MIT Press, Cambridge (2003)

Schrijver, A.: Theory of Linear and Integer Programming. Wiley, NewYork (1986)

Thomson, W.: Strategyproof allocation rules. Mimeo, December 2013 